\title{
HIGH ORDER ACCURATE METHODS FOR THE EVALUATION OF LAYER HEAT POTENTIALS*
}

\author{
JING-REBECCA LI $^{\dagger}$ AND LESLIE GREENGARD $\ddagger$
}

\begin{abstract}
We discuss the numerical evaluation of single and double layer heat potentials in two dimensions on stationary and moving boundaries. One of the principal difficulties in designing high order methods concerns the local behavior of the heat kernel, which is both weakly singular in time and rapidly decaying in space. We show that standard quadrature schemes suffer from a poorly recognized form of inaccuracy, which we refer to as "geometrically induced stiffness," but that rules based on product integration of the full heat kernel in time are robust. When combined with previously developed fast algorithms for the evolution of the "history part" of layer potentials, diffusion processes in complex, moving geometries can be computed accurately and in nearly optimal time.
\end{abstract}

Key words. heat potentials, moving boundaries, high order accuracy, quadrature, diffusion

AMS subject classifications. 31A10, 65R20, 65M12, 45D05, 45L05

DOI. $10.1137 / 080732389$

1. Introduction. In this paper, we consider some issues that arise in the solution of the heat equation in a nonstationary domain $\Omega_{T}$ with boundary $\Gamma_{T}$ :

$$
\begin{aligned}
\frac{\partial U}{\partial t}(\mathbf{x}, t)-\nabla^{2} U(\mathbf{x}, t) & =F(\mathbf{x}, t), & (\mathbf{x}, t) & \in \Omega_{T}=\prod_{\tau=0}^{T} \Omega(\tau), \\
U(\mathbf{x}, 0) & =U_{0}(\mathbf{x}), & \mathbf{x} \in \Omega(0), & \\
\alpha U(\mathbf{x}, t)+\beta \frac{\partial}{\partial n} U(\mathbf{x}, t) & =g(\mathbf{x}, t), & (\mathbf{x}, t) & \in \Gamma_{T}=\prod_{\tau=0}^{T} \Gamma(\tau) .
\end{aligned}
$$

That is, at each time $t, F(\mathbf{x}, t)$ is specified in $\Omega(t)$, the boundary condition (1.3) is imposed on $\Gamma(t)$, and $U(\mathbf{x}, t)$ is defined for $\mathbf{x} \in \Omega(t)$.

There are many methods for solving such problems, but we will restrict our attention to integral equation methods. Classical potential theory $[19,10]$ suggests seeking a solution of the form

$$
\begin{aligned}
U(\mathbf{x}, t)=\int_{\Omega(0)} & G(\mathbf{x}-\mathbf{y}, t) U_{0}(\mathbf{y}) d \mathbf{y}+\int_{0}^{t} \int_{\Omega(\tau)} G(\mathbf{x}-\mathbf{y}, t-\tau) F(\mathbf{y}, \tau) d \mathbf{y} d \tau \\
& +\int_{0}^{t} \int_{\Gamma(\tau)} G(\mathbf{x}-\mathbf{y}, t-\tau) \sigma(\mathbf{y}, \tau) d s_{\mathbf{y}} d \tau \\
& +\int_{0}^{t} \int_{\Gamma(\tau)} \frac{\partial}{\partial n_{\mathbf{y}}} G(\mathbf{x}-\mathbf{y}, t-\tau) \mu(\mathbf{y}, \tau) d s_{\mathbf{y}} d \tau
\end{aligned}
$$

${ }^{*}$ Received by the editors August 7, 2008; accepted for publication (in revised form) July 28, 2009; published electronically October 16, 2009.

http://www.siam.org/journals/sisc/31-5/73238.html

${ }^{\dagger}$ INRIA-Rocquencourt, Projet POEMS, Domaine de Voluceau - Rocquencour, 78153 Le Chesnay Cedex, France (jingrebecca.li@inria.fr).

${ }_{\ddagger}^{\ddagger}$ Courant Institute, New York University, New York, NY 10012 (greengard@courant.nyu.edu). This work was supported by the Applied Mathematical Sciences Program of the U.S. Department of Energy under contract DEFG0288ER25053. 
Here, $G(\mathbf{x}, t)$ is the fundamental solution of the heat equation in free space:

$$
G(\mathbf{x}, t)=(4 \pi t)^{-d / 2} \exp \left(\frac{-\|\mathbf{x}\|^{2}}{4 t}\right)
$$

$\mathbf{n}_{\mathbf{y}}$ is the unit outward normal to $\Gamma(t)$ at $\mathbf{y}, \frac{\partial}{\partial n_{\mathbf{y}}}$ denotes the derivative in the normal direction, $d s_{\mathbf{y}}$ is an element of arc length along the boundary, and $\sigma$ and $\mu$ are unknown surface densities defined on $\Gamma_{T}$. We will refer to the first integral in (1.4) as an initial potential, denoted by $G\left[U_{0}\right]$, to the second integral as a volume potential, denoted by $V[F]$, to the third integral as a single layer potential, denoted by $S[\sigma]$, and to the fourth integral as a double layer potential, denoted by $D[\mu]$. From the basic properties of the Green's function, the representation (1.4) clearly satisfies (1.1) and (1.2). It remains only to satisfy the boundary condition (1.3) for which we have allowed two unkown functions (the surface densities $\sigma$ and $\mu$ ). To avoid nonuniqueness issues, we make the simple choice of using the double layer potential alone for Dirichlet problems $(\beta=0)$ and the single layer potential alone for Neumann or Robin problems $(\beta \neq 0)$. Other methods, based on Green's identities, yield different formulations $[2,3,4,12]$.

We will require the following result $[19,10]$, which describes the limiting behavior of single and double layer potentials as $\mathbf{x} \rightarrow \Gamma(t)$, commonly referred to as jump conditions.

TheOREm 1. Let $D[\mu]$ denote the double layer potential on a sufficiently smooth boundary, and let $\mathbf{x}^{o}$ denote a point on $\Gamma(t)$. Then $D[\mu]$ satisfies the homogeneous heat equation $D[\mu](\mathbf{x}, 0)=0$ for $\mathbf{x} \notin \Gamma(0)$, and

$$
\begin{aligned}
& \lim _{\substack{\mathbf{x} \rightarrow \mathbf{x}^{o} \\
\mathbf{x} \in \Omega(t)}} D[\mu](\mathbf{x}, t)=-\frac{1}{2} \mu\left(\mathbf{x}^{o}, t\right)+D^{*}[\mu]\left(\mathbf{x}^{o}, t\right), \\
& \lim _{\substack{\mathbf{x} \rightarrow \mathbf{x}^{o} \\
\mathbf{x} \in c \Omega(t)}} D[\mu](\mathbf{x}, t)=\frac{1}{2} \mu\left(\mathbf{x}^{o}, t\right)+D^{*}[\mu]\left(\mathbf{x}^{o}, t\right),
\end{aligned}
$$

where

$$
D^{*}[\mu]\left(\mathbf{x}^{o}, t\right):=\int_{0}^{t} \int_{\Gamma(\tau)} \frac{\partial}{\partial n_{\mathbf{y}}} G\left(\mathbf{x}^{o}-\mathbf{y}, t-\tau\right) \mu(\mathbf{y}, \tau) d s_{\mathbf{y}} d \tau, \quad \mathbf{x}^{o} \in \Gamma(t),
$$

is weakly singular and $c \Omega(t)$ denotes the complement of $\Omega(t)$.

TheOREM 2. Let $S[\sigma]$ denote the single layer potential on a sufficiently smooth boundary, and let $\mathbf{x}^{o}$ denote a point on $\Gamma(t)$. Then $S[\sigma]$ satisfies the homogeneous heat equation $S[\sigma](\mathbf{x}, 0)=0$ for $\mathbf{x} \notin \Gamma(0)$, and

$$
\begin{aligned}
& \lim _{\substack{\mathbf{x} \rightarrow \mathbf{x}^{o} \\
\mathbf{x} \in \Omega(t)}} \frac{\partial}{\partial n_{\mathbf{x}^{o}}} S[\sigma](\mathbf{x}, t)=\frac{1}{2} \sigma\left(\mathbf{x}^{o}, t\right)+K^{*}[\sigma]\left(\mathbf{x}^{o}, t\right), \\
& \lim _{\substack{\mathbf{x} \rightarrow \mathbf{x}^{o} \\
\mathbf{x} \in \operatorname{co}(t)}} \frac{\partial}{\partial n_{\mathbf{x}^{o}}} S[\sigma](\mathbf{x}, t)=-\frac{1}{2} \sigma\left(\mathbf{x}^{o}, t\right)+K^{*}[\sigma]\left(\mathbf{x}^{o}, t\right),
\end{aligned}
$$

where

$$
K^{*}(\sigma)\left(\mathbf{x}^{o}, t\right):=\int_{0}^{t} \int_{\Gamma(\tau)} \frac{\partial}{\partial n_{\mathbf{x}^{o}}} G\left(\mathbf{x}^{o}-\mathbf{y}, t-\tau\right) \sigma(\mathbf{y}, \tau) d s_{\mathbf{y}} d \tau, \quad \mathbf{x}^{o} \in \Gamma(t),
$$

is weakly singular. 
The kernel of the single layer potential is also weakly singular, so that

$$
S[\sigma]\left(\mathbf{x}^{o}, t\right):=\int_{0}^{t} \int_{\Gamma(\tau)} G\left(\mathbf{x}^{o}-\mathbf{y}, t-\tau\right) \sigma(\mathbf{y}, \tau) d s_{\mathbf{y}} d \tau
$$

is well defined for $\mathbf{x}^{o} \in \Gamma(t)$.

In this paper, we restrict our attention to layer potentials and assume that the volume forcing term $F(\mathbf{x}, t)=0$. (Volume integrals are discussed, for example, in $[14,25]$.) For the Dirichlet problem $(\alpha=1$ and $\beta=0$ in (1.3)), taking the limit as a point $\mathbf{x} \in \Omega(t)$ approaches a point $\mathbf{x}^{o} \in \Gamma(t)$, we obtain the integral equation

$$
-\frac{1}{2} \mu\left(\mathbf{x}^{o}, t\right)+D^{*}[\mu]\left(\mathbf{x}^{o}, t\right)=g\left(\mathbf{x}^{o}, t\right)-G\left[U_{0}\right]\left(\mathbf{x}^{o}, t\right), \quad\left(\mathbf{x}^{o}, t\right) \in \Gamma_{T} .
$$

For the Neumann/Robin problem $(\beta \neq 0$ in $(1.3))$, we obtain

$$
\alpha S[\sigma]\left(\mathbf{x}^{o}, t\right)+\frac{\beta}{2} \sigma\left(\mathbf{x}^{o}, t\right)+\beta K^{*}[\sigma]\left(\mathbf{x}^{o}, t\right)=g\left(\mathbf{x}^{o}, t\right)-\alpha G\left[U_{0}\right]\left(\mathbf{x}^{o}, t\right)-\beta \frac{\partial}{\partial n_{\mathbf{x}^{o}}} G\left[U_{0}\right]\left(\mathbf{x}^{o}, t\right) .
$$

The integral equations in (1.12) and (1.13) are well-conditioned Volterra equations of the second kind and well suited to iterative solution. If we assume that some discretization rule in time is used with a time step of $\Delta t$, there is an obvious need for the efficient evaluation of $S[\sigma]$ and $D[\mu]$ on $\Gamma(t)$ at $t=n \Delta t, n=1,2,3, \ldots, N$. Since the Green's function is nonlocal in both space and time, it is straightforward to see that naive evaluation would require $O\left(N^{2} M^{2}\right)$ work, where $M$ is the number of points in the spatial discretization of the boundary. The initial potential would require $O\left(N M^{2}\right)$ work.

Fortunately, fast algorithms have been developed for layer potentials (and volume potentials) in both bounded and unbounded domains $[7,8,14,17,16,22,23]$. In this paper, we follow the approach of $[7,8]$. The first step in the development of these methods is the decomposition of layer potentials into a local part (the most recent contributions in time) and a history part (the most distant contributions in time):

$$
S[\sigma]\left(\mathbf{x}^{o}, t\right):=S_{L}[\sigma, \delta]\left(\mathbf{x}^{o}, t\right)+S_{H}[\sigma, \delta]\left(\mathbf{x}^{o}, t\right),
$$

where

$$
S_{L}[\sigma, \delta](\mathbf{x}, t):=\int_{t-\delta}^{t} \int_{\Gamma(\tau)} G(\mathbf{x}-\mathbf{y}, t-\tau) \sigma(\mathbf{y}, \tau) d s_{\mathbf{y}} d \tau
$$

and

$$
S_{H}[\sigma, \delta](\mathbf{x}, t):=\int_{0}^{t-\delta} \int_{\Gamma(\tau)} G(\mathbf{x}-\mathbf{y}, t-\tau) \sigma(\mathbf{y}, \tau) d s_{\mathbf{y}} d \tau
$$

The decompositions for $D[\mu], D^{*}[\mu], K^{*}[\mu]$ are defined in the analogous manner

$$
\begin{aligned}
D[\mu](\mathbf{x}, t) & :=D_{L}[\mu, \delta](\mathbf{x}, t)+D_{H}[\mu, \delta](\mathbf{x}, t), \\
D^{*}[\mu](\mathbf{x}, t) & :=D_{L}^{*}[\sigma, \delta](\mathbf{x}, t)+D_{H}^{*}[\sigma, \delta](\mathbf{x}, t), \\
K^{*}[\sigma, \delta](\mathbf{x}, t) & :=K_{L}^{*}[\sigma, \delta](\mathbf{x}, t)+K_{H}^{*}[\sigma, \delta](\mathbf{x}, t) .
\end{aligned}
$$

The fast algorithm is based essentially on three observations: (1) the heat kernel has an analytic expression as a Fourier integral, (2) for a fixed $\delta$, the decay of the high 
frequency components is very rapid, so that the integral can be computed over a finite range in the Fourier domain for any precision $\epsilon$, and (3) for each given frequency in that range, the Fourier transform satisfies a simple recursion in time, eliminating the explicit history dependence of the "physical space" representation. More precisely, if we define the Fourier transform in space of $S_{H}[\sigma, \delta](\mathbf{x}, t)$ by $\widehat{S_{H}}[\sigma, \delta](\mathbf{s}, t)$, then a straightforward calculation $[7,8]$ shows that each Fourier mode can be updated in time from the formula

$$
\begin{aligned}
\widehat{S_{H}}[\sigma, \delta](\mathbf{s}, t) & =e^{-\|\mathbf{s}\|^{2} \Delta t} \widehat{S_{H}}[\sigma, \delta](\mathbf{s}, t-\Delta t)+\Phi(\mathbf{s}, t, \Delta t, \delta), \\
\Phi(\mathbf{s}, t, \Delta t, \delta) & =\int_{t-\delta-\Delta t}^{t-\delta} e^{-\|\mathbf{s}\|^{2}(t-\tau)} \int_{\Gamma(\tau)} e^{i \mathbf{s} \cdot \mathbf{y}} \sigma(\mathbf{y}, \tau) d \mathbf{s}_{\mathbf{y}} d \tau .
\end{aligned}
$$

Likewise, if we define the Fourier transform in space of the double layer potential $D_{H}[\mu, \delta](\mathbf{x}, t)$ by $\widehat{D_{H}}[\mu, \delta](\mathbf{s}, t)$, then each Fourier mode can be updated in time from the formula

$$
\begin{aligned}
\widehat{D_{H}}[\mu, \delta](\mathbf{s}, t) & =e^{-\|\mathbf{s}\|^{2} \Delta t} \widehat{D_{H}}[\mu, \delta](\mathbf{s}, t-\Delta t)+\Phi_{D}(\mathbf{s}, t, \Delta t, \delta), \\
\Phi_{D}(\mathbf{s}, t, \Delta t, \delta) & =\int_{t-\delta-\Delta t}^{t-\delta} e^{-\|\mathbf{s}\|^{2}(t-\tau)} \int_{\Gamma(\tau)}\left(i \mathbf{s} \cdot \mathbf{n}_{\mathbf{y}}\right) e^{i \mathbf{s} \cdot \mathbf{y}} \mu(\mathbf{y}, \tau) d \mathbf{s}_{\mathbf{y}} d \tau .
\end{aligned}
$$

While the algorithm is somewhat intricate, the end result is that the history part can be evaluated accurately with only $O(N M \log M)$ work. Thus, to complete the evaluation of heat potentials, it remains only to evaluate the local parts $S_{L}[\sigma, \delta]\left(\mathbf{x}^{o}, t\right)$ and

$$
D_{L}^{*}[\mu, \delta]\left(\mathbf{x}^{o}, t\right)=\int_{t-\delta}^{t} \int_{\Gamma(\tau)} \frac{\partial}{\partial n_{\mathbf{y}}} G\left(\mathbf{x}^{o}-\mathbf{y}, t-\tau\right) \mu(\mathbf{y}, \tau) d \mathbf{s}_{\mathbf{y}} d \tau, \quad\left(\mathbf{x}^{o}, t\right) \in \Gamma_{T}
$$

for $\left(\mathbf{x}^{o}, t\right) \in \Gamma_{T}$. This calculation turns out to be surprisingly difficult.

Remark 1 . In some applications, the single or double layer potential is defined on a curve contained within a box on which periodic, Dirichlet, or Neumann boundary conditions are imposed. The history part is then represented by a Fourier series rather than a Fourier integral $[8,20]$. The local-in-time behavior of the heat kernel is the same as for the free space heat kernel, and the results concerning quadrature rules developed here apply with no essential change.

Remark 2. $K_{L}^{*}[\sigma, \delta](\mathbf{x}, t)$ can be treated in the same manner as its adjoint operator $D_{L}^{*}[\sigma, \delta](\mathbf{x}, t)$ and will not be considered separately.

Remark 3. Alternative methods for the rapid evaluation of layer heat potentials have been proposed, for example, in $[17,16,22,23]$. In $[17,16]$, the authors work with the Laplace transform in time of the heat kernel. In [22, 23], the authors develop a hierarchical space-time approximation scheme. While the treatment of the "history part" differs in each case, the local quadrature issues discussed here arise in essentially the same form and need to be addressed by all.

2. Asymptotics of local heat potentials in two dimensions. In order to study the "time" integrands of $S_{L}$ and $D_{L}^{*}$ in more detail, it is convenient to write them in the form

$$
S_{L}[\sigma, \delta]\left(\mathbf{x}^{o}, t\right):=\int_{t-\delta}^{t} \frac{1}{\sqrt{4 \pi(t-\tau)}} B_{S}[\sigma]\left(\mathbf{x}^{o}, t, \tau\right) d \tau,
$$

where

$$
B_{S}[\sigma]\left(\mathbf{x}^{o}, t, \tau\right)=\int_{\Gamma(\tau)} \frac{e^{-\frac{\left\|\mathbf{x}^{o}-\mathbf{y}\right\|^{2}}{4(t-\tau)}}}{\sqrt{4 \pi(t-\tau)}} \sigma(\mathbf{y}, \tau) d s_{\mathbf{y}},
$$


and

$$
D_{L}^{*}[\mu, \delta]\left(\mathbf{x}^{o}, t\right):=\int_{t-\delta}^{t} \frac{1}{\sqrt{4 \pi(t-\tau)}} B_{D}[\mu]\left(\mathbf{x}^{o}, t, \tau\right) d \tau
$$

where

$$
B_{D}[\mu]\left(\mathbf{x}^{o}, t, \tau\right)=\int_{\Gamma(\tau)} \frac{e^{-\frac{\left\|\mathbf{x}^{o}-\mathbf{y}\right\|^{2}}{4(t-\tau)}}}{4 \sqrt{\pi}(t-\tau)^{3 / 2}}\left(\mathbf{x}^{o}-\mathbf{y}\right) \cdot n_{\mathbf{y}} \mu(\mathbf{y}, \tau) d s_{\mathbf{y}}
$$

It is straightforward to show that (properly interpreted) $B_{S}[\sigma]\left(\mathbf{x}^{o}, t, \tau\right)$ and $B_{D}[\mu]\left(\mathbf{x}^{o}\right.$, $t, \tau)$ are both bounded as $\tau \rightarrow t$, so that the singularity in time of $S_{L}[\sigma, \delta]\left(\mathbf{x}^{o}, t\right)$ and $D_{L}^{*}[\mu, \delta]\left(\mathbf{x}^{o}, t\right)$ is indeed of the order $O(1 / \sqrt{t-\tau})$, as suggested in the expressions (2.1) and (2.3). As a result, one can simply expand $B_{S}[\sigma]\left(\mathbf{x}^{o}, t, \tau\right)$ and $B_{D}[\sigma]\left(\mathbf{x}^{o}, t, \tau\right)$ asymptotically as in $[8,15]$ to obtain the following.

THEOREM 3. Suppose that the coordinate system is translated and rotated so that $\mathbf{x}^{o}$ lies at the origin and the tangent at $\mathbf{x}^{o}$ is aligned with the $y_{1}$ axis where $\mathbf{y}=\left(y_{1}, y_{2}\right)$. Without loss of generality, let $\Gamma(\tau)$ be parametrized locally by $y_{1}=s$ and $y_{2}=y_{2}(s, \tau)$. Assume now that $y_{2}=y_{2}(s, \tau), \sigma(s, \tau)$, and $\mu(s, \tau)$ have the following Taylor expansions in $s$ and $t-\tau$ :

$$
\begin{aligned}
& y_{2}(s, \tau)=\gamma_{01}(t-\tau)+\frac{1}{2} \gamma_{20} s^{2}+\gamma_{11} s(t-\tau)+\frac{1}{2} \gamma_{02}(t-\tau)^{2}+\frac{1}{2} \gamma_{21} s^{2}(t-\tau) \\
&+\frac{1}{6} \gamma_{30} s^{3}+\frac{1}{2} \gamma_{12}(t-\tau)^{2} s+\frac{1}{6} \gamma_{03}(t-\tau)^{3}+\frac{1}{24} \gamma_{40} s^{4}+\cdots \\
& \sigma(s, \tau)=\sigma_{00}+\sigma_{10} s+\sigma_{01}(t-\tau)+\frac{1}{2} \sigma_{20} s^{2}+\sigma_{11} s(t-\tau)+\frac{1}{2} \sigma_{02}(t-\tau)^{2}+\cdots \\
& \mu(s, \tau)=\mu_{00}+\mu_{10} s+\mu_{01}(t-\tau)+\frac{1}{2} \mu_{20} s^{2}+\mu_{11} s(t-\tau)+\frac{1}{2} \mu_{02}(t-\tau)^{2}+\cdots
\end{aligned}
$$

Then

$$
\begin{aligned}
B_{S}[\sigma]\left(\mathbf{x}^{o}, t, \tau\right) & =\sigma_{00} \\
& +\left(\frac{\left(\gamma_{20}^{2}+\gamma_{01}^{2}-2 \gamma_{20} \gamma_{01}\right)}{4} \sigma_{00}+\left(\sigma_{01}-\sigma_{20}\right)\right)(t-\tau)+O\left((t-\tau)^{2}\right)
\end{aligned}
$$

and

$$
\begin{aligned}
B_{D}[\mu]\left(\mathbf{x}^{o}, t, \tau\right) & =\frac{1}{2}\left(\gamma_{20}-\gamma_{01}\right) \mu_{00} \\
& +\frac{1}{8}\left[\left(\gamma_{01}^{3}+\gamma_{01}^{2} \gamma_{20}-3 \gamma_{01} \gamma_{20}^{2}-15 \gamma_{20}^{3}-2 \gamma_{02}+4 \gamma_{21}+6 \gamma_{40}\right) \mu_{00}\right. \\
& \left.+16 \gamma_{30} \mu_{10}+\left(-4 \gamma_{01}+12 \gamma_{20}\right) \mu_{20}+\left(4 \gamma_{20}-4 \gamma_{01}\right) \mu_{01}\right](t-\tau) \\
& +O\left((t-\tau)^{2}\right)
\end{aligned}
$$

COROllary 1. The local part of the single layer potential has the asymptotic expansion [8]

$$
S_{L}[\sigma, \delta]\left(\mathbf{x}^{o}, t\right)=\left(\frac{\delta}{\pi}\right)^{1 / 2} \sigma\left(\mathbf{x}^{o}, t\right)+\delta^{3 / 2}\left(\frac{(\kappa-v)^{2} \sigma\left(\mathbf{x}^{o}, t\right)}{12 \sqrt{\pi}}+\frac{\left(\sigma_{t}-\sigma_{s s}\right)}{3 \sqrt{\pi}}\right)+O\left(\delta^{5 / 2}\right),
$$


where $\kappa$ denotes curvature and $v$ is the normal velocity at $\left(\mathbf{x}^{o}, t\right)$. The local part of the double layer potential has the asymptotic expansion

$$
D_{L}^{*}[\mu, \delta]\left(\mathbf{x}^{o}, t\right)=\delta^{1 / 2} \frac{(\kappa-v)}{2 \sqrt{\pi}} \mu\left(\mathbf{x}^{o}, t\right)+O\left(\delta^{3 / 2}\right) .
$$

Proof. The result follows from the preceding theorem, (2.1), (2.3), and the facts that $\sigma\left(\mathbf{x}^{o}, t\right)=\sigma_{00}, \sigma_{t}=\sigma_{01}, \sigma_{s s}=\sigma_{20}, \kappa=\gamma_{20}$, and $v=\gamma_{01}$.

Given the estimates in Theorem 3 and Corollary 1, it would appear to be quite efficient to use asymptotic formulas for the evaluation of $S_{L}, D_{L}^{*}$, and $K_{L}^{*}$. It is, of course, somewhat difficult to carry the analysis too far, since successive terms involve higher and higher order derivatives along the boundary. However, there is a more serious problem. Suppose the boundary $\Gamma$ has been discretized at $N$ equispaced points with spacing $\Delta x$ and that we are seeking to use a relatively large time step of the order $\Delta t \approx \Delta x$, as would typically be desired in an implicit marching scheme. The fast algorithm for the history part $[7,8]$ dictates that $\delta$ should be chosen of the order $O(\Delta x)$, so let us assume for the sake of simplicity that $\delta=\Delta x=\Delta t$. Since the heat kernel decays in space like $e^{-r^{2} / \delta}$, rather distant parts of the boundary must contribute to the value of the integral; more precisely, points that are $O(\sqrt{\delta})=O(\sqrt{\Delta x})$ away have a nontrivial effect. Assuming the boundary is of unit length, this means that the nearest $O(\sqrt{N})$ points should play a role in the evaluation of the layer potential. The asymptotic formulas, however, use only local or nearly local values and, therefore, can achieve the formal rate of convergence only when the influence of more distant boundary data is negligible. This implies, in turn, that $\delta=\Delta t$ should be of the order $O\left(\Delta x^{2}\right)$ (so that we are taking a standard explicit time step). Thus, while there is no stability issue in using asymptotics, there is an accuracy restriction that is quite severe. Clearly, in order to achieve high accuracy for large $\Delta t$ in a systematic fashion, we will require a different approach, based on a proper quadrature rule.

3. Quadrature methods for local heat potentials. A classical approach, widely used in the boundary element community [2,4], is to assume that the surface densities $\sigma$ and/or $\mu$ are piecewise constant over each time step and to compute the corresponding integrals analytically or semianalytically to some desired precision. For problems in one space dimension, where the densities are simply functions of time, Veerapaneni and Biros [24] and Huang, Lai, and Xiang [11] have recently developed elegant formalisms to extend this approach to arbitrarily high order accuracy. In [24], the authors use recurrence relations based on Chebyshev polynomial approximation of the density. In [11], the authors expand the integral of the full heat kernel (multiplied by monomials in time) in terms of the incomplete Gamma function. With these approaches, the quadrature errors are determined purely by the quality of approximation of the geometry and the surface densities. We will return to this approach in section 3.3. First, however, we consider what would appear to be a simpler and more attractive option.

3.1. Partial product integration. As noted in the preceding section, the representations (2.1) and (2.3) and the analysis of the functions $B_{S}[\sigma]$ and $B_{D}[\mu]$ in (2.2), (2.4) show that the time integrand has a simple inverse square root singularity. This suggests the use of a product integration method, such as the one introduced in [21]:

$$
S_{L}[\sigma, \delta]\left(\mathbf{x}^{o}, t\right)=\sqrt{\frac{\delta}{4 \pi}} \sum_{j=0}^{k} w_{j} B_{S}[\sigma]\left(\mathbf{x}^{o}, t, v_{j}\right)+e_{\delta}(k),
$$


where $e_{\delta}(k)$ is the error, the nodes $v_{j}=\frac{j}{k} \delta$ are equispaced, and the weights $w_{0}, \ldots, w_{k}$ are chosen so that

$$
\int_{t-\delta}^{t} \frac{1}{\sqrt{t-\tau}} g(\tau) d \tau=\sqrt{\delta} \sum_{j=0}^{k} w_{j} g\left(t-v_{j}\right)
$$

is exact for $g(v)$ a polynomial of degree $\leq k$. The error $e_{\delta}(k)$ is of the order

$$
e_{\delta}(k)=O\left(\delta^{k+3 / 2}\right)
$$

To see this, note that

$$
\begin{aligned}
e_{\delta}(k) & =\left|\int_{t-\delta}^{t} \frac{1}{\sqrt{t-\tau}} g(\tau) d \tau-\sum_{j=0}^{k} w_{j} g\left(t-v_{j}\right)\right| \\
& =\left|\int_{t-\delta}^{t} \frac{1}{\sqrt{t-\tau}}\left(g(\tau)-g_{I}(\tau)\right) d \tau\right| \\
& \leq \int_{t-\delta}^{t} \frac{1}{\sqrt{t-\tau}} d \tau\left\|g-g_{I}\right\|_{\infty} \leq C \delta^{k+3 / 2}\left\|g^{(k+1)}\right\|_{\infty} .
\end{aligned}
$$

Here $g_{I}$ denotes the polynomial of degree $k$ that interpolates $g$ at the given $k+1$ nodes.

We will refer to such schemes as partial product integration methods, since they take into account only part of the structure of the heat kernel. Unlike the asymptotic approaches, the integrals $B_{S}[\sigma]\left(\mathbf{x}^{o}, t, v_{j}\right)$ have the correct nonlocal data dependencies, so that there is no obvious drawback to using the formula. They have the important feature that the spatial integrals $\left(B_{S}\right.$ or $\left.B_{D}\right)$ are simply convolutions with a Gaussian and computable in linear time using the fast Gauss transform [9].

Variants of this approach can be developed for nonequispaced nodes on the interval $[t-\delta, t]$, based on Gauss-Jacobi quadrature or generalized versions of Gaussian quadrature $[1,13,18]$.

Unfortunately, partial product integration methods are not robust, especially in domains with high curvature. Like the asymptotic approaches, it turns out that these rules tend to require that $\delta$ be much smaller than the formal rate of convergence would suggest. We will illustrate this phenomenon, which we refer to as geometrically induced stiffness, with numerical examples in section 4 . First, however, we investigate this issue analytically.

3.2. Geometrically induced stiffness. To understand the effect of curvature on the evaluation of layer potentials, let us consider as a model problem the double layer potential on a parabola defined by

$$
\mathbf{y}=\left(\lambda, a \lambda^{2}\right), \quad-\infty<\lambda<\infty
$$


with a constant unit density $\mu=1$. We choose as the evaluation point the origin $\mathbf{x}^{o}=(0,0)$. Then,

$$
\begin{aligned}
& D_{L}^{*}[\mu, \Delta t]\left(\mathbf{x}^{o}, t\right)=\int_{t-\Delta t}^{t} \int_{\Gamma(\tau)} \frac{e^{-\frac{\|\mathbf{x}-\mathbf{y}(\tau)\|^{2}}{4(t-\tau)}}}{8 \pi(t-\tau)^{2}}\left[(\mathbf{x}-\mathbf{y}(\tau)) \cdot n_{\mathbf{y}(\tau)}\right] \mu(\mathbf{y}(\tau), \tau) d \sigma_{\mathbf{y}(\tau)} d \tau \\
& =\int_{0}^{\Delta t} \int_{-\infty}^{\infty} \frac{e^{-\frac{\left\|\left(\lambda, a \lambda^{2}\right)\right\|^{2}}{4 \tau}}}{8 \pi \tau^{2}}\left[\left(-\left(\lambda, a \lambda^{2}\right)\right) \cdot(2 a \lambda,-1)\right] d \lambda d \tau \\
& =\int_{0}^{a^{2} \Delta t} \int_{-\infty}^{\infty}-\frac{e^{-s^{2}} e^{-4 s^{4} w} s^{2}}{\pi} \frac{1}{\sqrt{w}} d s d w
\end{aligned}
$$

after the substitutions $s=\frac{\lambda}{\sqrt{4 \tau}}, w=a^{2} \tau$, and a little algebra.

OBSERVATION 1. Note that the parameter $a$ (which is one-half of the curvature at the origin) appears only in the limit of integration. Assume now that a quadrature rule in time using $k$ nodes has been constructed for $D_{L}^{*}[\mu, \Delta t]\left(\mathbf{x}^{o}, t\right)$. Then, if $a$ is increased by a factor of $m>1, \Delta t$ must be reduced by a factor of $m^{2}$ to preserve the same accuracy. On the other hand, to maintain spatial resolution as $a$ is increased to $m a$ requires only that $\Delta x$ be reduced by $1 / m$. In short, $\Delta t$ must be proportional to $\Delta x^{2}$ as the curvature increases.

More precisely, it follows from (2.4) and the change of variables above that

$$
B_{D}[\mu]\left(\mathbf{x}^{o}, t, \tau\right)=\frac{a}{8 \pi(t-\tau)^{3 / 2}} \int_{-\infty}^{\infty} \exp \left[-\lambda^{2} \frac{1+a^{2} \lambda^{2}}{4(t-\tau)}\right] \lambda^{2} d \lambda
$$

After changing varaibles $w=\lambda / \sqrt{t-\tau}$, the integral becomes

$$
B_{D}[\mu]\left(\mathbf{x}^{o}, t, \tau\right)=\frac{a}{\pi} \int_{-\infty}^{\infty} \exp \left[-\frac{w^{2}}{4}\left(1+a^{2}(t-\tau) w^{2}\right)\right] w^{2} d w .
$$

Since the integrand is smooth and in $L_{1}(\mathbb{R})$, one can interchange differentiation and integration, so that

$$
\frac{\partial^{k}}{\partial \tau^{k}} B_{D}[\mu]\left(\mathbf{x}^{o}, t, \tau\right)=\frac{a^{2 k+1}}{8 \pi} \int_{-\infty}^{\infty} \exp \left[-\frac{w^{2}}{4}\left(1+a^{2}(t-\tau) w^{2}\right)\right] \frac{w^{4 k+2}}{(-4)^{k}} d w .
$$

The integrals above converge and can be bounded independently of $a, t$, and $\tau$ :

$$
\int_{-\infty}^{\infty} \exp \left[-\frac{w^{2}}{4}\left(1+a^{2}(t-\tau) w^{2}\right)\right] \frac{w^{4 k+2}}{(-4)^{k}} d w \leq \int_{-\infty}^{\infty} \exp \left[-\frac{w^{2}}{4}\right] \frac{w^{4 k+2}}{(-4)^{k}} d w .
$$

Thus, $\left\|\frac{\partial^{k}}{\partial \tau^{k}} B_{D}[\mu]\right\|_{\infty}=O\left(a^{2 k+1}\right)$, and the quadrature error is bounded by

$$
e_{\delta}\left(B_{D}[\mu]\right) \leq C a^{2 k+3} \delta^{k+3 / 2}=C\left(a^{2} \delta\right)^{k+3 / 2}
$$

for some $C>0$, independent of $\delta$ and $a$. This proves the statement in the observation.

In some sense, this result should not be surprising - it stems from the natural scaling of $\Delta t$ and $\Delta x$ in the heat equation. This restriction is inherent in any partial product integration scheme.

The same phenomenon can be observed in evaluating a heat potential on a straight line with oscillatory data. For this, imagine that one seeks to compute the local part of 
a single layer potential on the $\mathrm{x}$-axis at the target $\mathbf{x}^{o}=(0,0)$ with $\sigma(y, \tau)=\sin (m y)$ :

$$
S_{L}[\sigma, \Delta t]\left(\mathbf{x}^{o}, t\right)=\int_{t-\Delta t}^{t} \int_{-\infty}^{\infty} \frac{e^{-\frac{\|y\|^{2}}{4(t-\tau)}}}{4 \pi(t-\tau)} \sin (m y) d y d \tau .
$$

The change of variables $\eta=m y, s=m^{2}(t-\tau)$ yields

$$
S_{L}[\sigma, \Delta t]\left(\mathbf{x}^{o}, t\right)=\frac{1}{m} \int_{0}^{m^{2} \Delta t} \int_{-\infty}^{\infty} \frac{e^{-\frac{\eta^{2}}{4 s}}}{4 \pi s} \sin (\eta) d \eta d s .
$$

As $m$ increases, $\Delta x \rightarrow \frac{\Delta x}{m}$ is sufficient to preserve spatial resolution, but $\Delta t \rightarrow \frac{\Delta t}{m^{2}}$ is necessary to preserve accuracy in time. With a slight abuse of language, we use the term geometrically induced stiffness to describe both phenomena.

It is worth repeating that neither formal order of accuracy or stability are at issue here - only the scaling required for a well-resolved scheme as the underlying physical problem gets harder and harder.

3.3. Full product integration in time. At this point, therefore, we return to the consideration of product integration in time using the full heat kernel. As mentioned earlier, this is considered a standard approach in the boundary element literature $[2,4]$ and has been carried out to high order in one dimension in [24, 11]. It was also suggested by Strain for the two-dimensional case [21] as a more robust approach than partial product integration for target points off the boundary.

It should be clear why these approaches avoid geometrically induced stiffness. Such quadratures are, by definition, exact for a piecewise polynomial approximation of the geometry and the surface densities. The complex coupling of spatial and temporal discretiation is avoided (at some analytic and computational expense).

Suppose first that the boundary is stationary - that is, $\Gamma(t)=\Gamma(0)$. The essential idea is to expand the density, say, $\sigma$, in the form

$$
\begin{aligned}
\sigma(\mathbf{x}, \tau)=\sigma_{0}(\mathbf{x}) & +(t-\tau) \sigma_{1}(\mathbf{x})+\frac{1}{2}(t-\tau)^{2} \sigma_{2}(\mathbf{x}) \\
+ & \cdots+\frac{1}{(k-1) !}(t-\tau)^{k-1} \sigma_{k-1}(\mathbf{x})+O\left((t-\tau)^{k}\right)
\end{aligned}
$$

and to change the order of integration.

The single layer potential $S_{L}$ then takes the form

$$
\begin{aligned}
S_{L}[\sigma, \delta](\mathbf{x}, t)=\frac{1}{4 \pi}[ & \int_{\Gamma} G_{0}(\mathbf{x}-\mathbf{y}) \sigma_{0}(\mathbf{y}) d s_{\mathbf{y}}+\int_{\Gamma} G_{1}(\mathbf{x}-\mathbf{y}) \sigma_{1}(\mathbf{y}) d s_{\mathbf{y}}+\ldots \\
& \left.+\frac{1}{(k-1) !} \int_{\Gamma} G_{k-1}(\mathbf{x}-\mathbf{y}) \sigma_{k-1}(\mathbf{y}) d s_{\mathbf{y}}\right]+O\left((t-\tau)^{k+1 / 2},\right.
\end{aligned}
$$

with the kernels $G_{k}(\mathbf{x})$ given by

$$
G_{k}(\mathbf{x})=\int_{t-\delta}^{t} e^{-\|\mathbf{x}\|^{2} / 4(t-\tau)}(t-\tau)^{k-1} d \tau .
$$


A straightforward calculation shows that

$$
G_{k}(\mathbf{x})= \begin{cases}\operatorname{Ei}\left(1, \frac{r^{2}}{4 \delta}\right), & k=0, \\ \frac{4 \delta e^{-\frac{r^{2}}{4 \delta}}-r^{2} \operatorname{Ei}\left(1, \frac{r^{2}}{4 \delta}\right)}{4}, & k=1, \\ \frac{16 \delta^{2} e^{-\frac{r^{2}}{4 \delta}}-4 r^{2} \delta e^{-\frac{r^{2}}{4 \delta}}+r^{4} \delta \operatorname{Ei}\left(1, \frac{r^{2}}{4 \delta}\right)}{32}, & k=2,\end{cases}
$$

where $\operatorname{Ei}(1, x)$ is the exponential integral function

$$
\operatorname{Ei}(1, x)=\int_{1}^{\infty} \frac{e^{-x t}}{t} d t
$$

Moving boundaries are a bit more complicated. The trick is to use the spatial domain at time $t$ for the entire interval $[t-\delta, t]$. We carry this out explicitly for the double layer potential:

$$
\begin{aligned}
D_{L}^{*}[\mu, \delta](\mathbf{x}, t) & =\int_{t-\delta}^{t} \int_{\Gamma(\tau)} \frac{e^{-\frac{\|\mathbf{x}-\mathbf{y}(\tau)\|^{2}}{4(t-\tau)}}}{8 \pi(t-\tau)^{2}}\left[(\mathbf{x}-\mathbf{y}(\tau)) \cdot n_{\mathbf{y}(\tau)}\right] \mu(\mathbf{y}(\tau), \tau) d \sigma_{\mathbf{y}(\tau)} d \tau \\
& =\int_{\Gamma(t)} \int_{t-\delta}^{t} \frac{e^{-\frac{\|\mathbf{x}-\mathbf{y}(t)\|^{2}}{4(t-\tau)}}}{8 \pi(t-\tau)^{2}}[h(\mathbf{y}, \tau)] d \tau d \sigma_{\mathbf{y}(t)}
\end{aligned}
$$

where

$$
h(\mathbf{y}, \tau):=e^{-\frac{\|\mathbf{y}(t)-\mathbf{y}(\tau)\|^{2}}{4(t-\tau)}} e^{-\frac{2(\mathbf{x}-\mathbf{y}(t)) \cdot(\mathbf{y}(t)-\mathbf{y}(\tau))}{4(t-\tau)}}\left[(\mathbf{x}-\mathbf{y}(\tau)) \cdot n_{\mathbf{y}(\tau)}\right] \mu(\mathbf{y}(\tau), \tau) \frac{d \sigma_{\mathbf{y}(\tau)}}{d \sigma_{\mathbf{y}(t)}} .
$$

It is this function that is expanded as a Taylor series in time:

$$
\begin{aligned}
h(\mathbf{y}, \tau)=h_{0}(\mathbf{y}) & +(t-\tau) h_{1}(\mathbf{y})+\frac{1}{2}(t-\tau)^{2} h_{2}(\mathbf{y}) \\
& +\cdots+\frac{1}{(k-1) !}(t-\tau)^{k-1} h_{k-1}(\mathbf{y})+O\left((t-\tau)^{k}\right) .
\end{aligned}
$$

Changing the order of integration yields

$$
\begin{aligned}
D_{L}^{*}[\mu, \delta](\mathbf{x}, t)=\frac{1}{8 \pi}[ & \int_{\Gamma} G_{-1}(\mathbf{x}-\mathbf{y}) h_{0}(\mathbf{y}) d s_{\mathbf{y}}+\int_{\Gamma} G_{0}(\mathbf{x}-\mathbf{y}) h_{1}(\mathbf{y}) d s_{\mathbf{y}}+\ldots \\
& \left.+\frac{1}{(k-1) !} \int_{\Gamma} G_{k-2}(\mathbf{x}-\mathbf{y}) h_{k-1}(\mathbf{y}) d s_{\mathbf{y}}\right]+O\left((t-\tau)^{k+1 / 2}\right) .
\end{aligned}
$$

Note that the double layer involves the kernel $G_{-1}$, which can be computed explicitly from (3.4):

$$
G_{-1}(\mathbf{x})=\frac{e^{-\frac{r^{2}}{4 \delta}}}{\pi r^{2}}
$$

It remains only to consider the nature of the spatial singularities in the evaluation of (3.9). The apparent $\frac{1}{r^{2}}$ singularity in $G_{-1}$ does not cause problems because it is canceled by the term $(\mathbf{x}-\mathbf{y}(\tau)) \cdot n_{\mathbf{y}(\tau)}$ in $h_{0}(\mathbf{y})$. More precisely,

$$
(\mathbf{x}-\mathbf{y}(\tau)) \cdot n_{\mathbf{y}(\tau)} \sim-2 \gamma_{02}(\mathbf{x}, t) r^{2},
$$

where $\gamma_{02}(\mathbf{x}, t)$ is the curvature at $(\mathbf{x}, t)$. 
The spatial singularity that remains to be dealt with is the logarithmic singularity in the exponential integral function:

$$
\operatorname{Ei}(1, z)=-\gamma-\ln (z)+z-\frac{z^{2}}{4}+O\left(z^{3}\right) .
$$

This is easily handled using a variety of quadratures [1, 13, 18], all of which are capable of high order accuracy for integrands of the form

$$
f(x) \ln (|x|)+g(x),
$$

assuming only that $f(z)$ and $g(z)$ are smooth. Unfortunately, each of the integrals in (3.3) or (3.9) involves a different kernel, so that a suitable fast algorithm is required for each.

Finally, note that this approach is suitable for target points off the boundary with no essential change (as mentioned above).

4. Numerical examples. In order to verify the preceding analysis and to test the actual performance of the methods under discussion, we have implemented them all (in Fortran).

Remark 4. All spatial integrals are computed in this section to high precision, so that the errors in the examples come only from the time quadrature.

For our first example, we consider as a domain the moving ellipse

$$
\begin{aligned}
& y_{1}(\lambda, t)=2 \cos (\lambda)+1.5 t, \\
& y_{2}(\lambda, t)=\sin (\lambda),
\end{aligned}
$$

We evaluate the local part of the double layer potential $D_{L}^{*}[\mu, \Delta t](\mathbf{x}, t)$ with density $\mu \equiv 1$ using six different schemes:

1. first order asymptotics [ASYMP1];

2. five point partial product integration using equispaced nodes [PP-EQ5];

3. five point partial product integration using Gauss-Jacobi nodes and weights [5] tuned for a square root singularity [PP-GJ5];

4. five point generalized Gaussian quadrature [18] tuned for a square root singularity [PP-GGQ5];

5. full product integration carried out to fourth order in the Taylor series [FP4].

In Figure 4.1, we plot the errors in $D_{L}^{*}[\mu, \Delta t](\mathbf{x}, t)$ at $t=1$ for several values of $\Delta t$. The reference solution is obtained numerically using a twentieth order Gauss-Jacobi rule on a very fine grid.

It can be seen that $\mathrm{FP} 4$ gives the best results, with the immediate onset of the expected order of convergence. Formally, PP-EQ5 and PP-GGQ5 have convergence order 5.5, while PP-GJ5 has convergence order 10.5. Among these partial product integration strategies, PP-GGQ5 gives the best results, but the main thing to note is the delay in the onset of the formal behavior.

For our second example, we test the order of convergence of the partial product integration schemes on the parabola for which we carried out a mathematical analysis in section 3.2. We consider the methods ASYMP1, PP-EQ2 (two equispaced nodes), and PP-GJ2 (two Gauss-Jacobi nodes), with formal orders of convergence 1.5, 2.5, and 4.5 , respectively. The boundary is given by

$$
\begin{aligned}
& y_{1}(\lambda, t)=\lambda, \\
& y_{2}(\lambda, t)=a \lambda^{2}, \quad 0 \leq \lambda \leq 2 \pi
\end{aligned}
$$




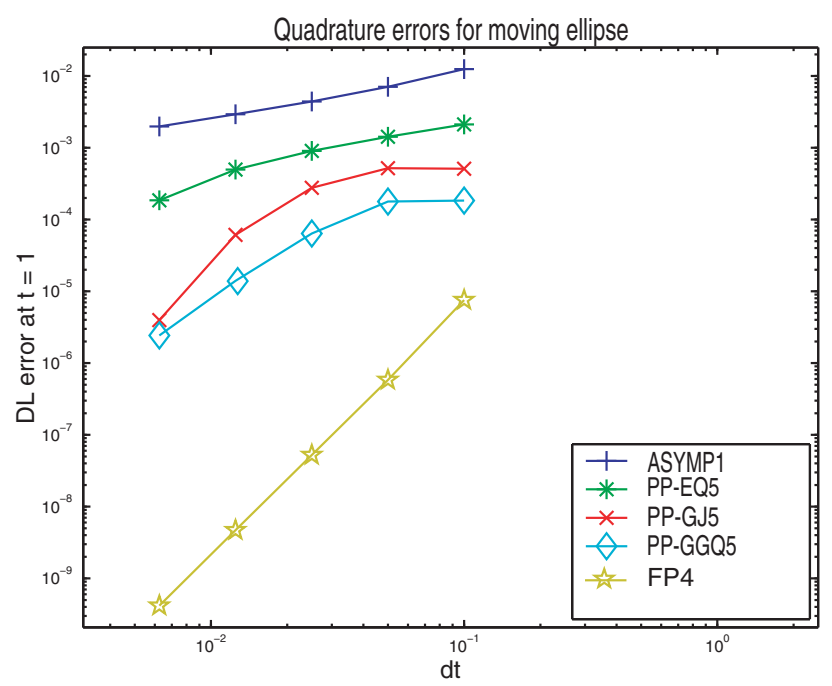

FIG. 4.1. Comparison of quadrature methods for the double layer potential on a moving ellipse with constant density.

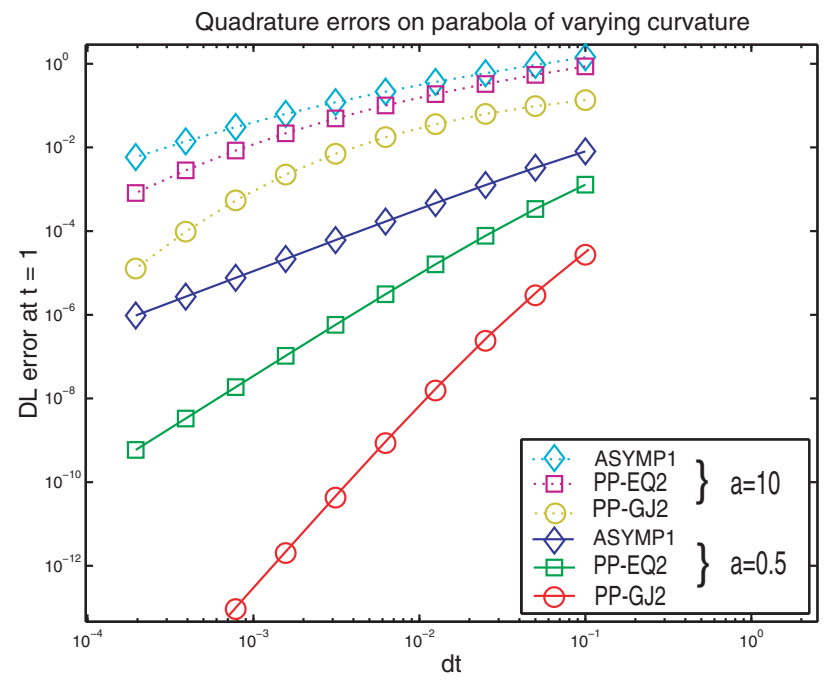

FIG. 4.2. Comparison of quadrature methods for the double layer potential on parabolas with different curvatures.

for which $a$ is one-half of the curvature at the origin. We assume the density is constant $\mu \equiv 1$. In Figure 4.2 we plot the resulting errors for $a=0.5$ and $a=10$. Over the range of $\Delta t$ plotted, the orders of convergence 1.5, 2.5, and 4.5 are apparent when the curvature is small $(a=0.5)$. When the curvature is high, however, the onset of convergence is significantly delayed, as expected. We don't plot the results for full product integration (since it is exact for a density that is constant in time).

For our final example, we use the same moving ellipse as in the first example. We study the performance of the full product integration schemes carried out to two and four terms in the Taylor expansion. The resulting schemes FP2 and FP4 have formal 


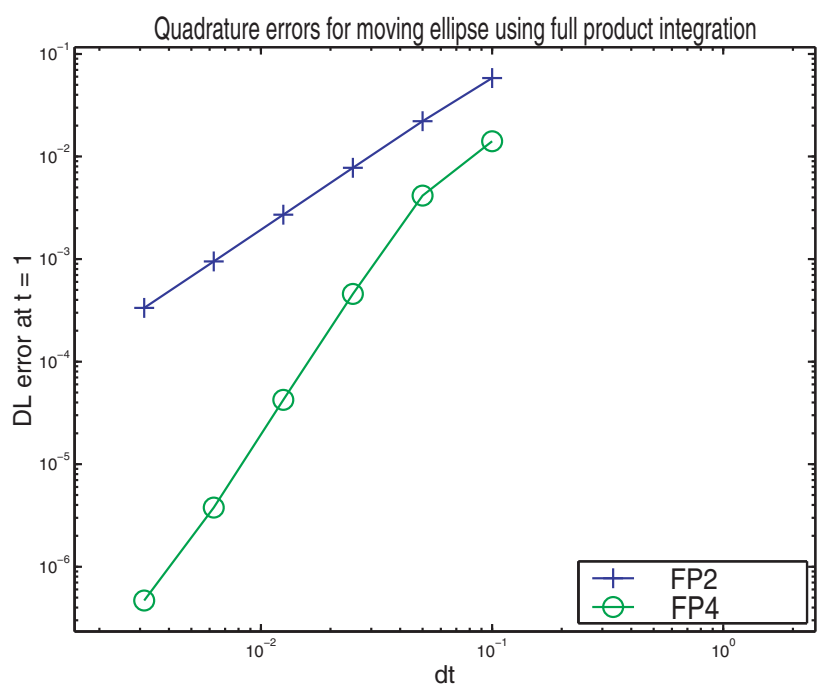

FIG. 4.3. Comparison of quadrature methods for the double layer potential on a moving ellipse with a variable density.

convergence orders of 1.5 and 3.5 , respectively. Rather than use a constant density, we now let

$$
\mu\left(y_{1}, y_{2}, t\right)=\cos \left(y_{1} t\right)+\sin (10 t) .
$$

The results are shown in Figure 4.3.

5. Conclusions. The numerical evaluation of single and double layer heat potentials in two dimensions is a surprisingly complicated task. We have shown that neither asymptotics nor partial product integration schemes are robust-both being highly sensitive to geometric features. We have investigated this loss of the expected order of convergence analytically and refer to it as "geometrically induced stiffness," since it forces the time step $\Delta t$ to be proportional to $\Delta x^{2}$ under certain conditions. This is a slight misnomer, since in the integral equation context it manifests itself as a form of inaccuracy rather than a loss of stability. As expected, full product integration of the heat kernel results in robust schemes, since the error comes only from the approximations made in representing the geometry and the single or double layer densities themselves. We have used a Taylor series formalism here, which works best for orders of accuracy up to six or so. For higher order, it has recently been shown [26] that the one-dimensional recursive approach of [24] applies in higher spatial dimensions as well.

In order to construct optimal time algorithms, fast algorithms are needed to compute the spatial convolution integrals that arise in (3.3) and (3.9). This can be accomplished, for example, by generalized fast multipole methods [6, 27]. In their recent work [26], Veerapaneni and Biros have shown that careful use of recursion reduces the number of different kernels for which fast algorithms are required. Finally, the local quadrature schemes developed here need to be combined with previously developed fast algorithms for the evolution of the "history part" of layer potentials. The full scheme, including volume sources, will allow for the accurate modeling of diffusion processes in complex, moving geometries and will be reported at a later date. 
Acknowledgments. The authors thank the anonymous referees for several suggestions that improved the presentation of paper.

\section{REFERENCES}

[1] B. K. Alpert, Hybrid Gauss-trapezoidal quadrature rules, SIAM J. Sci. Comput., 20 (1999), pp. 1551-1584.

[2] C. A. Brebbia, J. C. F. Telles, And L. C. Wrobel, Boundary Element Techniques, SpringerVerlag, Berlin, 1984.

[3] M. Costabel, Time-dependent problems with the boundary integral equation, in Encyclopedia of Computational Mechanics, E. Stein, R. de Borst, and T. J. R. Hughes, eds., John Wiley \& Sons, New York, 2004, pp. 703-721.

[4] G. F. Dargush and P. K. BanerJee, Application of the boundary element method to transient heat conduction, Internat. J. Numer. Methods Engrg., 31 (1991), pp. 1231-1247.

[5] P. J. Davis and P. Rabinowitz, Methods of Numerical Integration, Academic Press, San Diego, 1984.

[6] Z. Gimbutas and V. Rokhlin, A generalized fast multipole method for nonoscillatory kernels, SIAM J. Sci. Comput., 24 (2003), pp. 796-817.

[7] L. Greengard and P. Lin, Spectral approximation of the free-space heat kernel, Appl. Comput. Harmon. Anal., 9 (2000), pp. 83-97.

[8] L. Greengard and J. Strain, A fast algorithm for the evaluation of heat potentials, Comm. Pure Appl. Math., 43 (1990), pp. 949-963.

[9] L. Greengard and J. Strain, The fast Gauss transform, SIAM J. Sci. Statist. Comput., 12 (1991), pp. 79-94.

[10] R. B. Guenther and J. W. Lee, Partial Differential Equations of Mathematical Physics and Integral Equations, Prentice Hall, Englewood Cliffs, NJ, 1988.

[11] J. Huang, M.-C. LaI, And Y. XIANG, An integral equation method for epitaxial step-flow growth simulations, J. Comput. Phys., 216 (2006), pp. 724-743.

[12] M. T. Ibanez And H. Power, An efficient direct BEM numerical scheme for phase change problems using fourier series, Comput. Methods Appl. Mech. Engrg., 10 (2002), pp. 687720 .

[13] S. KAPUR AND V. RokHLIN, High-order corrected trapezoidal quadrature rules for singular functions, SIAM J. Numer. Anal., 34 (1997), pp. 1331-1356.

[14] J.-R. Li And L. GReEngard, On the numerical solution of the heat equation I: Fast solvers in free space, J. Comput. Phys., 226 (2007), pp. 1891-1901.

[15] P. Lin, On the Numerical Solution of the Heat Equation in Unbounded Domains, Ph.D. thesis, New York University, New York, 1993.

[16] Ch. Lubich And A. Ostermann, Runge-Kutta methods for parabolic equations and convolution quadrature, Math. Comp., 60 (1993), pp. 105-131.

[17] Ch. Lubich And R. Schneider, Time discretization of parabolic boundary integral equations, Numer. Math., 63 (1992), pp. 455-481.

[18] J. Ma, V. Rokhlin, AND S. WANDZuRA, Generalized Gaussian quadrature rules for systems of arbitrary functions, SIAM J. Numer. Anal., 33 (1996), pp. 971-996.

[19] W. Pogorzelski, Integral Equations and Their Applications, Pergamon Press, Oxford, 1966.

[20] J. A. Sethian And J. Strain, Crystal growth and dendritic solidification, J. Comput. Phys., 98 (1992), pp. 231-253.

[21] J. Strain, Fast potential theory II. Layer potentials and discrete sums, J. Comput. Phys., 99 (1992), pp. 251-270.

[22] J. Strain, Fast adaptive methods for the free-space heat equation, SIAM J. Sci. Comput., 15 (1994), pp. 185-206.

[23] J. TAusch, A fast method for solving the heat equation by layer potentials, J. Comput. Phys., 224 (2007), pp. 956-969.

[24] S. K. VeErapaneni ANd G. Biros, A high-order solver for the heat equation in $1 D$ domains with moving boundaries, SIAM J. Sci. Comput., 29 (2007), pp. 2581-2606.

[25] S. K. Veerapaneni and G. Biros, The Chebyshev fast Gauss and nonuniform fast Fourier transforms and their application to the evaluation of distributed heat potentials, J. Comput. Phys., 227 (2008), pp. 7768-7790.

[26] S. K. Veerapaneni and G. Biros, Arbitrary-order Accurate Schemes for Computing Layer Heat Potentials, University of Pennsylvania, preprint, 2008.

[27] L. Ying, G. Biros, AND D. Zorin, A kernel-independent adaptive fast mulitpole method in two and three dimensions, J. Comput. Phys., 196 (2004), pp. 591-626. 